\title{
Path Planning for Multiple Robots: An Alternative Duality Approach ${ }^{\dagger}$
}

\author{
Nader Motee $^{1}$, Ali Jadbabaie ${ }^{2}$, and George Pappas ${ }^{2}$
}

\begin{abstract}
We propose an optimization-based framework to find multiple fixed length paths for multiple robots that satisfy the following constraints: (i) bounded curvature, (ii) obstacle avoidance, (iii) and collision avoidance. By using polygonal approximation techniques, we show that path planning problem for multiple robots under various constraints and missions, such as curvature and obstacle avoidance constraints as well as rendezvous and maximal total area coverage, can be cast as a nonconvex optimization problem. Then, we propose an alternative dual formulation that results in no duality gap. We show that the alternative dual function can be interpreted as minimum potential energy of a multi-particle system with discontinuous spring-like forces. Finally, we show that using the proposed duality-based framework, an approximation of the minimal length path planning problem (also known as Dubins' problem) in presence of obstacles can be solved efficiently using primal-dual interior-point methods.
\end{abstract}

\section{INTRODUCTION}

The path planning problem for a robot in an environment with obstacles has been an active research area in robotics and control communities in past two decades [1], [2]. The major trends have been focused on nonholonomic kinematic path planning problems. The primary challenge to plan a collision-free path for robots is nonholonomic constrains that limit steering and locomotion. This introduces restrictions on the way they are able to change directions. The Dubins' classical problem [3] has been the core of many of research results in this area where it explains how to characterize shortest bounded-curvature paths for a robot with given initial and terminal points and tangents. There are several results related to shortest paths among obstacles without curvature constraints [4]-[9]. Canny and Reif showed that the problem, among general polyhedral obstacles, is NPhard in the three-dimensional Euclidean space [10]. These results partially motivated researchers to develop various approximate methods to solve the path planning problem [11], [12], [15], [16]. There are several polynomial algorithms for the two-dimensional case. The two-dimensional shortest path problem has been formulated mainly in two different settings: semi-algebraic obstacles [4], [13] and polygonal obstacles [6], [7], [14]. Nevertheless, the existing algorithms are incomplete in the sense that they may not provide a solution even if one exists.

\footnotetext{
1 The author is with the Control and Dynamical Systems Department, California Institute of Technology, 1200 E. California Blvd, Pasadena, CA 91125 USA (motee@cds.caltech.edu).

2 The authors are with the Department of Electrical and Systems Engineering and GRASP Laboratory, University of Pennsylvania, 200 South 33rd Street, Philadelphia, PA 19104 USA ( $\{$ jadbabai, pappasg\}@seas. upenn.edu) .

$\dagger$ This work is supported by ONR MURI HUNT N00014-08-1-0696 and ARO CTA MAST W911NF-08-2-0004.
}

In this paper, our goal is to propose a scalable optimization-based framework for solving the boundedcurvature path planning problem for multiple robots with multiple missions in presence of moving obstacles. We assume that motion trajectories of obstacles are known. First, we consider the path planning problem for a single robot. Then, we extend our results to handle multi-robot path planning problems. Our approach is based on polygonal approximation of a continuous curve in the plane [27]. A path connecting the initial and final positions of a robot can be approximated by finitely many waypoints. This approximation can be arbitrarily improved by increasing the number of waypoints. In this setting, we can relax the boundedcurvature and collision-free constraints by verifying the constraints only at these waypoints. This relaxation results in a finite-dimensional formulation of the path planning problem as a nonconvex feasibility problem. Every feasible solution to the relaxed problem is an approximate boundedcurvature and collision-free path for the robot. Moreover, we show that various task planning for multiple robots such as rendezvous and maximal area coverage can be cast as nonconvex optimization problems.

The difficulty of solving such nonconvex feasibility problems is twofold. First, since there are equality constraints in these formulations, the problem is not directly amenable to standard optimization algorithms such as interior-point method. Second, even if we have an efficient algorithm to handle equality constraints, finding a feasible initial point is not easy. Indeed, any feasible solution is a solution of the path planning problem and finding a feasible initial is equivalent to solving the path planning problem. Therefore, nonconvex feasibility optimization problems arising in various task planning problems for multiple robots are inherently difficult and cannot be tackled directly.

On the other hand, a large class of nonconvex optimization problems can be efficiently solved by primal-dual interiorpoint methods only if one can find a dual formulation that has no duality gap [17]-[26]. Duality provides a theoretical foundation for many optimization algorithms to directly solve optimization problems as well as to obtain a lower bounds on the optimal value of the problem. However, for noncovex optimization problems the traditional duality theory leads to duality gaps and that most of the primal-dual algorithms cannot be directly applied to solve the problem. In Section III, we propose an alternative duality formulation that results in no duality gap for a general class of optimization problems. The main feature of the proposed algorithm (see Table I) is that it initiates the search for an optimal solution from small penalties and increases them incrementally until 
the duality gap is zero.

In Section IV, we show that the alternative dual function has an interesting dynamical system interpretation. We show that the value of the alternative dual function can be viewed as minimum of the potential energy of all spring-like forces in a multi-particle system. Through this connection, we may solve the alternative dual problem by using the method developed in [27]. It is shown that each waypoint in the optimization problem can be treated as a moving particle in the plane. One can define interaction spring-like forces between the particles such that: (i) the set of equilibria of the system contains all feasible solutions of the optimization problem, and (ii) the corresponding multi-particle system is asymptotically stable. In [27], it is shown that by applying some specific type of discontinuous spring-like forces, the net force on each particle is equal to zero if and only if these particles are representing a feasible path. In other words, for every initial condition the trajectory of the system always converges to a feasible path for the robot.

In Section $\mathrm{V}$, we show that minimal length path planning problem (also known as Dubins' problem) in presence of obstacles (possibly nonconvex) can be formulated in alternative duality framework. We show that the duality gap for minimal path planning problem is zero and that it can be solved efficiently using tools developed in Section III.

\section{Task Planning for Multiple Vehicles}

In this section, we show that various task planning problems for multiple robots can be approximated by polygonal curves and formulated as nonconvex optimization problems. Let denote $\|$.$\| to be the 2$-dimensional Euclidean norm and $\mathcal{I}_{n}=\{1, \ldots, n\}$ and $\mathbf{D}(c, r)=\{x \mid\|x-c\| \leq r\}$. Our approach is based on discrete approximation of a continuous curve of length $L$ with maximum curvature $\kappa_{\max }$ using finite number of vertices. Consider a polygonal curve $\hat{\chi}=$ $\overline{x_{0} x_{1} \ldots x_{n}}$ represented by its ordered vertices $x_{0}, x_{1}, \ldots, x_{n} \in$ $\mathbb{R}^{2}$ where $\overline{x_{i} x_{i+1}}$ is the line segment connecting $x_{i}$ to $x_{i+1}$. Under some mild assumptions, for a given error bound $\epsilon>0$, one can always find points $\left\{x_{0}, x_{1}, \ldots, x_{n}\right\}$, for a large number $n>0$, such that

$$
|\operatorname{Length}(\hat{\chi})-L|<\epsilon,
$$

where

$$
\operatorname{Length}(\hat{\chi})=\sum_{i=1}^{n}\left\|x_{i}-x_{i-1}\right\| .
$$

Without loss of generality, we may assume that all points $x_{i}$ are equidistant. Therefore, it follows that

$$
l=\left\|x_{i}-x_{i-1}\right\| \simeq \frac{L}{n} .
$$

for all $i \in \mathcal{I}_{n}$. We can impose curvature constraint on $\hat{\chi}$ by imposing some norm constraints on the vertices [28]. If we assume that $l \ll \frac{1}{\kappa_{\max }}$, then we can impose the discrete curvature constraint $\kappa_{i} \leq \kappa_{\max }$ through the following norm constraints on the vertices

$$
\left\|x_{i-1}-x_{i+1}\right\| \geq l \sqrt{4-\kappa_{\max }^{2} l^{2}}=\eta
$$

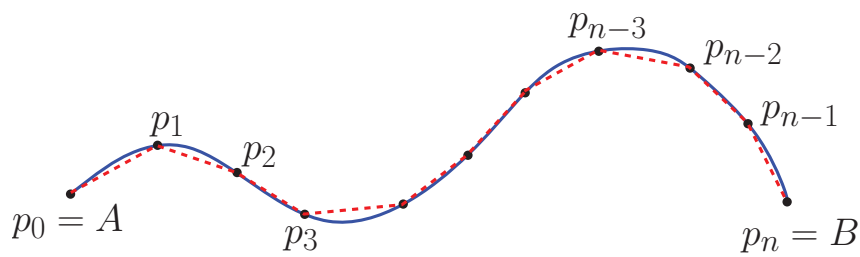

Fig. 1: Approximation of a smooth curve $\chi$ by an equidistant polygonal curve $\hat{\chi}=\overline{x_{0} x_{1} \ldots x_{n}}$.

for all $i \in \mathcal{I}_{n-1}$. In the following subsections, we show that one can formulate various task planning problems for multiple robots as feasibility optimization problems.

\section{A. Single-Vehicle Path Planning in Presence of Obstacles}

The goal of this subsection is to find a fixed-length bounded curvature trajectory for a robot with given initial and final configurations in an environment with obstacles. We assume that the Dubins robot is traveling with a constant speed $V$ and that can traverse a distance $L$ in $\frac{L}{V}$ time unites. Suppose that there are $M$ moving obstacle with known motion patterns in the environments. At any time instant $t$, each obstacle is assumed to be represented by a disk $\mathbf{D}\left(c_{j}(t), r_{j}(t)\right)=\left\{x \mid\left\|x-c_{j}(t)\right\| \leq r_{j}(t)\right\}$. We also assume that these disks are not overlapping for all time. Let $\kappa_{\max }>0$ be the maximum allowable curvature and $A, B \in \mathbb{R}^{2}$ the initial and final points. Then the path planning problem consists of finding a curve $\chi:[0, T] \rightarrow \mathbb{R}^{2}$ (parameterized by time) such that

(i) $\quad \chi(0)=A$ and $\chi(T)=B$.

(ii) $\quad \kappa(t) \leq \kappa_{\max }$ for all $t \in[0, T]$.

(iii) $\quad \chi(t) \cap \mathbf{D}\left(c_{j}(t), r_{j}(t)\right)=\emptyset$ holds for all $t \in[0, T]$ and $j \in \mathcal{I}_{M}$.

Note that $\kappa(t)$ is the curve curvature at time $t$. One can see that $\chi$ is a fixed length curve of length $L=V T$. We refer to the third condition as the obstacle avoidance constraint. The second condition guarantees a bounded curvature curve. An arbitrarily fine approximation of the path planning problem can be obtained by using polygonal approximation of $\chi$. The obstacle avoidance constraint implies to find waypoints $x_{0}, \ldots x_{n}$ such that

$$
\forall i \in \mathcal{I}_{n}, j \in \mathcal{I}_{M} \quad\left\|x_{i}-c_{j}\left(t_{i}\right)\right\| \geq r_{j}\left(t_{i}\right)
$$

in which $t_{i}$ is the time instant at which the robot visits waypoint $x_{i}$. By incorporating obstacle avoidance constraints (4) and curvature constraints (3), the path planning can be formulated as the following optimization problem

$\min _{\mathbf{x}} 0$

subject to: $x_{0}=A, x_{n}=B$

$$
\begin{array}{ll}
\left\|x_{i}-x_{i-1}\right\|=l, & i \in \mathcal{I}_{n} \\
\left\|x_{i-1}-x_{i+1}\right\| \geq \eta, & i \in \mathcal{I}_{n-1} \\
\left\|x_{i}-c_{j}\left(t_{i}\right)\right\| \geq r_{j}\left(t_{i}\right), & i \in \mathcal{I}_{n-1}, j \in \mathcal{I}_{M} .
\end{array}
$$


where $\mathbf{x}=\left(x_{0}^{T}, x_{1}^{T}, \ldots, x_{n}^{T}\right)^{T}$ and $l$ and $\eta$ are defined in (2) and (3). It is straightforward to verify that (5) is a nonconvex optimization problem. In the following, we extend this formulation for multiple robots.

\section{B. Multi-Vehicle Path Planning in Presence of Obstacles}

A similar formulation can be easily extended to multiple robots. We assume that there are $N$ robots with constant speeds $V_{k}$ traveling distances $L_{k}$ for all $k \in \mathcal{I}_{N}$. The initial and final points for each robot is given and are denoted, respectively, by $A_{k}$ and $B_{k}$ for all $k \in \mathcal{I}_{N}$. The corresponding waypoints for robot $k$ is represented by $\left(x_{0}^{k}, x_{1}^{k}, \ldots, x_{n_{k}}^{k}\right)$ where $n_{k}$ is the number of waypoints for robot $k$. For each robot, the curvature constraint can be guaranteed by imposing constraint (3) on the corresponding waypoints. In order to avoid collision between these robots, we must guarantee that any two robots, say $p$ and $q$, are not going to arrive at a waypoint simultaneously. In other words, if there exist waypoints $x_{i_{p}}^{p}$ and $x_{i_{q}}^{q}$ for which

$$
\left|\frac{L_{p}}{n_{p} V_{p}} i_{p}-\frac{L_{q}}{n_{q} V_{q}} i_{q}\right| \leq \epsilon_{\mathrm{time}}
$$

then the following constraint has to be imposed on the corresponding waypoints

$$
\left\|x_{i_{p}}^{p}-x_{i_{q}}^{q}\right\| \geq \epsilon_{\text {position }}
$$

for some time-error $\epsilon_{\text {time }}>0$ and position-error $\epsilon_{\text {position }}>$ 0 due to discretization. All indices satisfying (6) can be calculated before hand. We denote the index set of such pairs of waypoints as $\mathcal{I}_{\text {collision }}^{p, q}$ and $\mathbf{x}^{k}=\left(x_{0}^{k T}, x_{1}^{k T}, \ldots, x_{n_{k}}^{k T}\right)^{T}$. Therefore, the path planning problem for multiple robots in the presence of moving obstacles can be formulated as the following nonconvex feasibility problem

$$
\min _{\mathbf{x}^{1}, \ldots, \mathbf{x}^{N}} 0
$$

$$
\begin{aligned}
& \text { subject to: } x_{0}^{k}=A_{k}, \quad x_{n_{k}}^{k}=B_{k} \\
& \left\|x_{i}^{k}-x_{i-1}^{k}\right\|=l_{k} \text {, } \\
& \left\|x_{i-1}^{k}-x_{i+1}^{k}\right\| \geq \eta_{k} \text {, } \\
& i \in \mathcal{I}_{n_{k}} \\
& \left\|x_{i}^{k}-c_{j}\left(t_{i}^{k}\right)\right\| \geq r_{j}\left(t_{i}^{k}\right), \quad i \in \mathcal{I}_{n_{k}-1}, j \in \mathcal{I}_{M} \\
& \forall k \in \mathcal{I}_{N} \\
& \left\|x_{i_{p}}^{p}-x_{i_{q}}^{q}\right\| \geq \epsilon_{\text {position }}, \quad\left(i_{p}, i_{q}\right) \in \mathcal{I}_{\text {collision }}^{p, q} \\
& \forall p, q \in \mathcal{I}_{N}
\end{aligned}
$$

As one can see, problem (8) contains $N$ copies of (5) for each robots plus the collision avoidance constraints among the robots. This is also a nonconvex feasibility problem.

\section{Multi-Vehicle Path Planning with Rendezvous}

We assume that there are $N$ robots traveling with constant speeds that should rendezvous at a given time $0<$ $T_{\text {rendezvous }}<T$. For each robot $k$, we denote the index of the waypoint corresponding to the rendezvous time $T_{\text {rendezvous }}$ by $i_{k}$. These points should satisfy the following inequality

$$
\left|\frac{L_{k}}{n_{k} V_{k}} i_{k}-T_{\text {rendezvous }}\right| \leq \epsilon_{\text {time }}
$$

for all $k \in \mathcal{I}_{N}$ and for some time-error $\epsilon_{\text {time }}>0$ due to discretization. For each robot, the corresponding index satisfying (9) can be calculated. Then the rendezvous condition is that at time $T_{\text {rendezvous }}$ all robots has to be in a $\epsilon_{\text {position }}$ neighborhood of other robots, i.e.,

$$
\left\|x_{i_{p}}^{p}-x_{i_{q}}^{q}\right\| \leq \epsilon_{\text {position }}
$$

for all $p, q \in \mathcal{I}_{N}$. Therefore, the path planning problem for multiple robots with rendezvous can be formulated as a nonconvex feasibility problem in the form of (8) in which the collision avoidance constraints are replaced with the rendezvous constraints (10).

\section{Path Planning for Multiple Vehicles with Maximal Area Coverage}

The goal is to generate trajectories for multiple robots that maximize coverage while satisfying hard constraints such as collision avoidance and specifications on initial and final positions. We assume that each robot is equipped with a sensor which has a fixed sensing radius. Since trajectories are fixed length, one way to maximize the overall coverage is to minimize the intersection of field of view of waypoints.

We assume that $x_{i}^{k}$, the $i^{\text {th }}$ waypoint of robot $k$, has field of view $\mathbf{D}\left(x_{i}^{k}, r_{i}^{k}\right)$. In order to maximize the total coverage, we minimize the intersection of all discs corresponding to waypoints from different robots by imposing the following constraints

$$
\left\|x_{i}^{p}-x_{j}^{q}\right\| \geq r_{i}^{p}+r_{j}^{q}
$$

for all $p, q \in \mathcal{I}_{N}, i \in \mathcal{I}_{n_{p}}$, and $j \in \mathcal{I}_{n_{q}}$.

Therefore, the path planning problem for multiple robots with maximal area coverage can be cast as a nonconvex feasibility problem of the form (8) with additional coverage maximization constraints (11).

The difficulty of solving the above mentioned feasibility problems is twofold. First, since there are equality constraints in all the formulations, these problems are not directly amenable to an interior-point method. Second, even if we have an efficient algorithm to handle equality constraints, finding a feasible initial point is not easy. Indeed, any feasible solution is a solution of the path planning problem and finding a feasible initial is equivalent to solving (15). Therefore, nonconvex feasibility optimization problems arising in various task planning problems for multiple robots are inherently difficult and cannot be tackled directly. In the following, we propose an alternative dual formulation that helps to solve problem this class of problems as an unconstrained problem by using standard optimization tools such primal-dual interior-point methods.

\section{Alternative Duality for General Nonconvex PROBLEMS}

Duality provides a theoretical foundation for many optimization algorithms to directly solve optimization problems as well as to obtain a lower bound on the optimal value of the problem. However, for noncovex optimization problems the traditional duality theory leads to duality gaps and that 
most of the primal-dual algorithms cannot be directly applied to solve the problem. In this section, we introduce the alternative duality formulation for the following class of optimization problems that results in no duality gap,

$$
\begin{array}{ll}
\min _{x \in \mathcal{X}} f(x) & \\
\text { subject to: } & h(x)=0, \\
& g(x) \leq 0
\end{array}
$$

in which $\mathcal{X}$ is a compact subset of $\mathbb{R}^{n}, f: \mathbb{R}^{n} \rightarrow \mathbb{R}$ a differentiable function, and $h(x)=\left(h_{1}(x), \ldots, h_{m}(x)\right)^{T}$ and $g(x)=\left(g_{1}(x), \ldots, g_{r}(x)\right)^{T}$. We assume that $h_{i}: \mathbb{R}^{n} \rightarrow$ $\mathbb{R}$ and $g_{j}: \mathbb{R}^{n} \rightarrow \mathbb{R}$ are continuous functions on $\mathcal{X}$, but not necessarily differentiable. Let define the set of feasible points of (12) as follows $\mathcal{F}:=\{x \in \mathcal{X} \mid h(x)=0, g(x) \leq 0\}$.

Definition 1: The alternative dual function for the primal problem (12) is defined as

$$
\Delta(\mu, \lambda)=\min _{x \in \mathcal{X}} \Theta(x, \mu, \lambda)
$$

where

$$
\Theta(x, \mu, \lambda)=f(x)+\sum_{i=1}^{m} \mu_{i}\left|h_{i}(x)\right|+\sum_{j=1}^{r} \lambda_{j} g_{j}^{+}(x) .
$$

The alternative dual problem can be cast as

$$
d^{*}=\max _{\mu, \lambda \succeq 0} \Delta(\mu, \lambda) .
$$

We assume that (12) has a unique global minimizer denoted by $x^{*}$. The corresponding minimum value is denoted by $p^{*}$,

$$
p^{*}=f\left(x^{*}\right)=\min _{x \in \mathcal{F}} f(x) .
$$

In the following theorem, we show that the duality gap between a primal problem (12) and its corresponding alternative dual problem (18) is zero, i.e., $d^{*}=p^{*}$.

Theorem 1: Suppose that $x^{*}$ is the unique global minimizer of (12). Then, there exist finite vectors $\mu^{*} \succeq 0$ and $\lambda^{*} \succeq 0$ such that

$$
f\left(x^{*}\right)=\min _{x \in \mathcal{X}} \Theta(x, \mu, \lambda) \quad \text { for all } \quad \mu \succeq \mu^{*}, \quad \lambda \succeq \lambda^{*} .
$$

Moreover, the duality gap is zero, i.e., $p^{*}=d^{*}$.

Proof: We refer to [27] for a proof.

In general, the primal problem (12) can be a nonconvex problem. Theorem 1 implies that for large enough dual parameters $\mu_{i}$ and $\lambda_{i}$ solving the unconstraint optimization problem (13) results in finding the unique global minimum of the primal problem (12). Using this result, we can develop a suitable primal-dual algorithm that is amenable to interiorpoint methods.

The main feature of the proposed algorithm in Table I is that it initiates the search for an optimal solution from small penalties and increases them incrementally until the duality gap is zero. In the following section, we show that there is an interesting connection between the alternative dual formulation and elastic multi-particle systems [28].

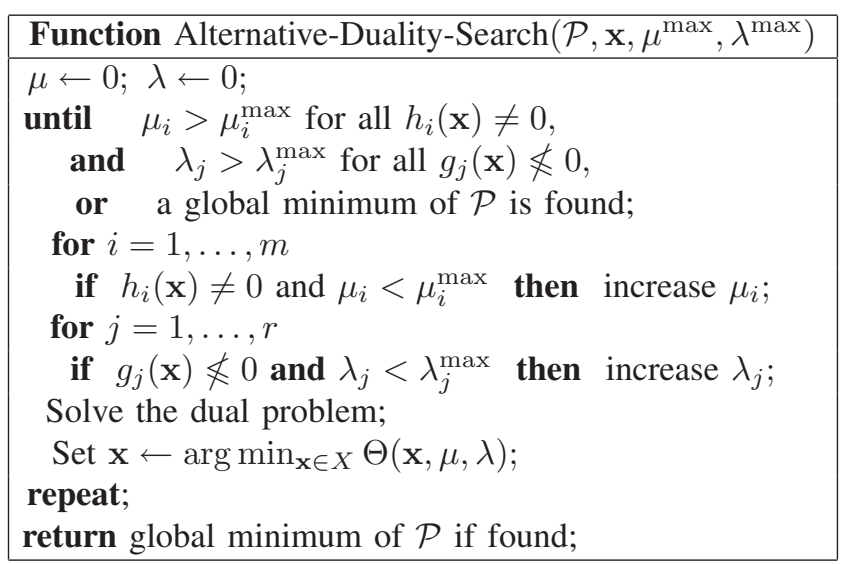

TABLE I: Implementation of the Alternative Duality Method

\section{A DYNAMICAL SYSTEM INTERPRETATION OF ALTERNATIVE DUALITY}

In the Section II, we showed that various task planning problems for multiple robots can be formulated as a nonconvex feasibility problem with different types of norm constraints on the waypoints. For the sake of simplicity, we focus our attention on the following single-robot path planning problem in presence of an obstacle which is characterized as a disk $\mathbf{D}(c, r)$,

$$
\begin{array}{lll}
\underset{\mathbf{x}}{\min } 0 & & \\
\text { subject to: } & x_{0}=A, \quad x_{n}=B & \\
& \left\|x_{i}-x_{i-1}\right\|=l, & i \in \mathcal{I}_{n} \\
& \left\|x_{i-1}-x_{i+1}\right\| \geq \eta, & i \in \mathcal{I}_{n-1} \\
& \left\|x_{i}-c\right\| \geq r, & i \in \mathcal{I}_{n-1}
\end{array}
$$

where $\mathbf{x}=\left(x_{0}^{T}, x_{1}^{T}, \ldots, x_{n}^{T}\right)^{T}$ and $l$ and $\eta$ are defined in (2) and (3). The following analysis can be directly extended to the other path planning problems introduced in subsections II-B, II-C, and II-D. In the following, we show that the alternative dual formulation has an interesting dynamical system interpretation. We show that the dual alternative function for (15) can be interpreted as the total potential energy of an elastic multi-particle system with discontinuous spring-like forces. We consider the alternative dual function for (15)

$$
\Delta(\mu, \lambda, \gamma)=\min _{\mathbf{x}} \Theta(\mathbf{x}, \mu, \lambda, \gamma)
$$

where

$$
\begin{aligned}
\Theta(\mathbf{x}, \mu, \lambda, \gamma)= & \sum_{i=1}^{n} \mu_{i}\left|\left\|x_{i}-x_{i-1}\right\|-l\right| \\
& +\sum_{i=1}^{n-1} \lambda_{i} \max \left\{0, \eta-\left\|x_{i-1}-x_{i+1}\right\|\right\} \\
& +\sum_{i=1}^{n-1} \gamma_{i} \max \left\{0, r-\left\|x_{i}-c\right\|\right\}
\end{aligned}
$$

in which $x_{0}=A$ and $x_{n}=B$, and for all $\mu=$ $\left(\mu_{1}, \ldots, \mu_{n}\right)^{T} \in \mathbb{R}^{n}, \lambda=\left(\lambda_{1}, \ldots, \lambda_{n-1}\right)^{T} \in \mathbb{R}^{n-1}$, and 
$\gamma=\left(\gamma_{1}, \ldots, \gamma_{n-1}\right)^{T} \in \mathbb{R}^{n-1}$. Moreover, the alternative dual problem can be defined as follows

$$
\begin{aligned}
& \max \Delta(\mu, \lambda, \gamma) \\
& \text { subject to: } \quad \mu \succeq 0, \quad \lambda, \gamma \succeq 0 .
\end{aligned}
$$

The waypoints $x_{i} \in \mathbb{R}^{2}$ can be viewed as spatial position of point mass particles moving on the plane. Let $m_{i}$ be the mass of particle $i$ with position $x_{i}$. We can apply a net force $F_{i}$ to control the dynamics of the particle according to Newton's second law

$$
m_{i} \ddot{x}_{i}=F_{i}
$$

where $i=0,1, \ldots, n$. We define discontinuous spring-like forces $f_{i j}$ among the particles as follows. For each $i$, the spring-like force $f_{i(i+1)}$ is applied to impose the equidistance constraints

$$
f_{i(i+1)}(z)=\left\{\begin{array}{clc}
\mu_{i} & \text { if } & z>l \\
0 & \text { if } & z=l \\
-\mu_{i} & \text { if } & z<l
\end{array},\right.
$$

the spring-like force $f_{(i-1)(i+1)}$ is employed to impose the curvature constraint on the waypoints

$$
f_{(i-1)(i+1)}(z)=\left\{\begin{array}{cll}
0 & \text { if } & z>\eta \\
-\lambda_{i} & \text { if } & z \leq \eta
\end{array},\right.
$$

and the spring-like force $f_{i \mathcal{O}}$ is defined to impose the obstacle avoidance constraint

$$
f_{i \mathcal{O}}(z)=\left\{\begin{array}{cll}
0 & \text { if } & z>r \\
-\gamma_{i} & \text { if } & z \leq r
\end{array} .\right.
$$

Finally, one can impose the following hard constraints

$$
x_{0}=A \quad \text { and } \quad x_{n}=B
$$

on particles 0 and $n$ by assuming that $m_{0}, m_{n}$ are arbitrarily large. In other words, two heavy masses are concentrated at points $A$ and $B$ and that their positions are fixed.

It is straightforward to verify that the alternative dual function (16) is indeed equal to the total potential energy of a multi-particle system with spring-like forces (20)-(22), i.e.,

$\Theta(\mathbf{x}, \mu, \lambda, \gamma)=\sum_{i=1}^{n} \mathbf{W}_{i(i-1)}\left(\left\|x_{i}-x_{i-1}\right\|\right)$

$+\sum_{i=1}^{n-1} \mathbf{W}_{(i-1)(i+1)}\left(\left\|x_{i-1}-x_{i+1}\right\|\right)+\sum_{i=1}^{n-1} \mathbf{W}_{i \mathcal{O}}\left(\left\|x_{i}-c\right\|\right)$

where

$$
\mathbf{W}_{i j}(\alpha)=\int_{\alpha_{0}}^{\alpha} f_{i j}(z) d z
$$

and $\alpha_{0}$ is a zero of spring-like function $f_{i j}$.

The right hand of (23) is the total potential energy of all springs in the multi-particle system. Thus, the dual function (16) is the minimum potential energy of all springs which is parameterized by the magnitude of spring-like forces $\mu$, $\lambda$, and $\gamma$. In [28], it is shown that if the magnitude of the spring-like forces are chosen appropriately, then the set of all stable equilibria of the dynamical systems (19) is equal to the set of all feasible solutions of nonconvex optimization problem (15). In the following theorem, $\mathbf{e}_{i j}=\frac{x_{j}-x_{i}}{\left\|x_{i}-x_{j}\right\|}$, $\mathbf{e}_{i \mathcal{O}}=\frac{c-x_{i}}{\left\|c-x_{i}\right\|}$, and all spring-like forces corresponding to undefined indices are assumed to be zero.

Theorem 2: Consider the multi-particle dynamical system (19) with net force

$$
\begin{aligned}
& F_{i}=f_{(i-1) i}\left(\left\|x_{i-1}-x_{i}\right\|\right) \mathbf{e}_{(i-1) i}+ \\
& f_{i(i+1)}\left(\left\|x_{i}-x_{i+1}\right\|\right) \mathbf{e}_{i(i+1)}+f_{(i-2) i}\left(\left\|x_{i-2}-x_{i}\right\|\right) \mathbf{e}_{(i-2) i} \\
& +f_{i(i+2)}\left(\left\|x_{i}-x_{i+2}\right\|\right) \mathbf{e}_{i(i+2)}+f_{i \mathcal{O}}\left(\left\|x_{i}-c\right\|\right) \mathbf{e}_{i \mathcal{O}}-v \dot{x}_{i}
\end{aligned}
$$

where the spring-like forces are discontinuous and defined as in (20)-(22) and $v>0$ is a constant. Then for almost all initial conditions, the trajectories of the multi-particle dynamical system (19) asymptotically converge to an equilibrium. Furthermore, a feasible solution of problem (15) is a locally asymptotically stable equilibrium of the multi-particle dynamical system (19) if all the corresponding spring-like forces are equal to zero.

Proof: We refer to [27] for a proof.

The importance of the result of Theorem 2 is that it provides a reliable approximation method to solve feasibility problem (15). We refer to [27] for further discussions on a nonsmooth dynamical system approach to solve (15).

\section{Minimal Length Path Planning in Presence of OBSTACLES}

In this section, we extend our duality-based framework to find an approximate solution for a minimal length path planning problem (also known as Dubins' problem) in presence of obstacles (possibly nonconvex). For simplicity of notations, we explain the problem for a single robot in presence of an obstacle. The extension of the problem formulation to multiple robots and obstacles under various missions is straightforward. Our objective is to find a polygonal curve $\hat{\chi}=\overline{x_{0} x_{1} \ldots x_{n}}$ with minimal length such that $\hat{\chi}$ satisfies: (1) $x_{0}=A$ and $x_{n}=B$, (2) the obstacle avoidance constraints, (3) and the curvature constraints. We assume that the obstacle is defined as $\mathcal{O}=\left\{x \in \mathbb{R}^{2} \mid g(x)>0\right\}$. We assume that $g(A)<0$ and $g(B)<0$. We assume that $\mathcal{O}$ is a bounded set, but it can be a nonconvex set. The minimal length path planning in presence of obstacle $\mathcal{O}$ can be cast as the following optimization problem:

$$
\begin{aligned}
& p^{*}=\min _{\mathbf{x}} \sum_{i=1}^{n}\left\|x_{i}-x_{i-1}\right\| \\
& \text { subject to: }\left\|x_{i-1}-x_{i+1}\right\| \geq \eta, \quad i \in \mathcal{I}_{n-1} \\
& g\left(x_{i}\right) \leq 0, \quad i \in \mathcal{I}_{n-1}
\end{aligned}
$$

where $x_{0}=A, x_{n}=B$, and $\eta$ is defined in (3). We can also impose initial and terminal tangents by fixing the position of waypoints $x_{1}=C$ and $x_{n-1}=D$ where $\overline{C A}$ and $\overline{B D}$ are initial and final desired tangents. The alternative dual function for (24) is given by

$$
\Delta(\lambda, \gamma)=\min _{\mathbf{x}} \Theta(\mathbf{x}, \lambda, \gamma)
$$


where

$$
\begin{aligned}
\Theta(\mathbf{x}, \lambda, \gamma)= & \sum_{i=1}^{n}\left\|x_{i}-x_{i-1}\right\| \\
& +\sum_{i=1}^{n-1} \lambda_{i} \max \left\{0, \eta-\left\|x_{i-1}-x_{i+1}\right\|\right\} \\
& +\sum_{i=1}^{n-1} \gamma_{i} \max \left\{0, g\left(x_{i}\right)\right\}
\end{aligned}
$$

where $x_{0}=A, x_{n}=B$, and $\lambda, \gamma \in \mathbb{R}^{n-1}$. The alternative dual problem can be defined as follows

$$
\begin{aligned}
d^{*}= & \max \Delta(\lambda, \gamma) \\
& \text { subject to: } \quad \lambda \succeq 0, \quad \gamma \succeq 0
\end{aligned}
$$

According to Theorem 1, for large enough dual parameters the duality gap between the minimal length path planning problem (24) and its corresponding dual problem (27) is zero.

\section{CONCLUSION}

We proposed a framework to obtain an arbitrarily fine relaxation of a path planning problem for multiple robots as a nonconvex feasibility problem. We showed that rendezvous and maximal area coverage problems for multiple robots can also be cast as nonconvex feasibility problems using this framework. Then, we proposed an alternative dual formulation with no duality gap. This new dual formulation enables us to tackle the resulting nonconvex optimization problems by primal-dual interior point algorithms. We also showed that the Dubins' problem can be cast as a nonconvex optimization problem and can be solved efficiently by using alternative dual formulation. Our preliminary studies shows that the alternative duality requires to incur smaller penalties in order to achieve zero duality gap. This is practically important as smaller function values results in well-conditioned primaldual algorithms.

\section{REFERENCES}

[1] J. C. Latombe. Robot Motion Planning. Kluwer Academic Publishers, 1991

[2] S. M. LaValle. Planning Algorithms. Cambridge University Press, 2006

[3] L. E. Dubins, On curves of minimal length with a constraint on average curvature, and with prescribed initial and terminal positions and tangents, Amer. J. Math., vol. 79, no. 3, pp. 497-516, 1957

[4] D. Grigoriev and A. Slissenko. Polytime algorithm for the shortest path in a homotopy class amidst semi-algebraic obstacles in the plane. In ISSAC 98: Proceedings of the 1998 international symposium on Symbolic and algebraic computation, pages 1724. ACM Press, 1998.

[5] G. Heinzinger, P. Jacobs, J. Canny, and B. Paden. Time-optimal trajectories for a robotic manipulator: A provably good approximation algorithm. In Proceedings IEEE International Conference on Robotics and Automation, pages 150155, Cincinnati, OH, 1990.

[6] J. Hershberger and S. Suri. An optimal algorithm for Euclidean shortest paths in the plane. SIAM J. Computing, 28:22152256, 1999.

[7] T. Lozano-Perez and M. A. Wesley. An algorithm for planning collision-free paths among polyhedral obstacles. Communications of the ACM, 22(10):560570, 1979.

[8] J. S. B. Mitchell. Planning Shortest Paths. PhD thesis, Stanford University, 1986.

[9] N. J. Nilsson. A mobile automaton: An application of artificial intelligence techniques. In 1st International Joint Conference on Artificial Intelligence, pages 509520, 1969.
[10] J. Canny and J. Reif. New lower bound techniques for robot motion planning problems. In Proc. IEEE Conf. on Foundations of Computer Science, pages 4960, 1987.

[11] Joonsoo Choi, Jurgen Sellen, and Chee-Keng Yap. Approximate euclidean shortest path in 3-space. In Annual ACM Symposium on Computational Geometry, pages 4148, New York, NY, USA, 1994. ACM.

[12] C. H. Papadimitriou. An algorithm for shortest-path planning in three dimensions. Information Processing Letters, 20(5):259263, 1985.

[13] J. Heintz, T. Krick, A. Slissenko, and P. Solerno. Search for shortest path around semialgebraic obstacles in the plane. J. Math Sciences, 70(4):19441949, 1994.

[14] J. Hershberger and J. Snoeyink. Computing minimum length paths of a given homotopy class. Computational geometry, 4:6397, 1994.

[15] P. Jacobs and J. Canny. Planning smooth paths for mobile robots. In Proceedings of the IEEE International Conference on Robotics and Automation, 1989.

[16] H. Wang and P. K. Agarwal. Approximation algorithms for curvatureconstrained shortest paths. In Proceedings of the seventh annual ACMSIAM symposium on Discrete algorithms, Philadelphia, PA, USA, 1996.

[17] D.P. Bertsekas. Distributed dynamic programming. Trans. Autom. Control, AC-27(3):610616, 1982.

[18] R. T. Rockafellar. Augmented Lagrangian multiplier functions and duality in nonconvex programming. SIAM J. Control Optim., 12:268285, 1974.

[19] J. V. Burke. Calmness and exact penalization. SIAM J. Control Optim., 29:493497, 1991

[20] J. V. Burke. An exact penalization viewpoint of constrained optimization. SIAM J. Control Optim. 29: 968998, 1991.

[21] A. M. Rubinov, B. M. Glover, X. Q. Yang. Decreasing functions with applications to penalization. SIAM J. Optim., 10: 289313, 1999.

[22] X. Q. Yang, X. X. Huang. A nonlinear Lagrangian approach to constraint optimization problems. SIAM J. Optim., 11:11191144, 2001.

[23] Z. Q. Luo, J. S. Pang. Error bounds in mathematical programming. Math. Program. Ser. B, 88(2), 2000.

[24] A. Nedi'c, A., A. Ozdaglar. A geometric framework for nonconvex optimization duality using augmented Lagrangian functions. J. Glob. Optim., 40(4):545573, 2008

[25] J. S. Pang. Error bounds in mathematical programming. Math. Program., 79:299332, 1997.

[26] D. P. Bertsekas. Nonlinear Programming, Athena Scientific, 1995.

[27] N. Motee, A. Ahmadzadeh, A. Jadbabaie, and G. Pappas. Elastic Multi-Particle Systems for Multi-Vehicle Path Planning. IEEE Transactions on Automatic Control, January 2010. Submitted.

[28] A. Ahmadzadeh, N. Motee, A. Jadbabaie, and G. Pappas. MultiVehicle Path Planning in Dynamically Changing Environments. Proceedings of the IEEE International Conference on Robotics and Automation, Kobe, Japan, May 2009. 